\title{
Chiral Soliton Lattice in Chiral Magnetic Crystal $\mathrm{CrNb}_{3} \mathrm{~S}_{6}$
}

\author{
Y. TOGAWA ${ }^{\text {a) }}$, T. KOYAMA ${ }^{\text {b) }}$, S. MORI ${ }^{\text {b) }}$, Y. KOUSAKA $^{\text {() }}$, J. AKIMITSU $^{\text {() }}$, \\ S. NISHIHARA ${ }^{\text {d) }}$, K. INOUE ${ }^{\text {d) }}$, A. S. OVCHINNIKOV ${ }^{\text {e) }}$, and J. KISHINE ${ }^{\text {f) }}$
}

a) Nanoscience \& Nanotechnology Research Center, Osaka Prefecture University, 1-2 Gakuencho, Naka, Sakai, Osaka 599-8570, Japan

b) Department of Materials Science, Osaka Prefecture University, 1-2 Gakuencho, Naka-ku, Sakai, Osaka, 599-8531, Japan

c) Department of Physics and Mathematics, Aoyama Gakuin University, Sagamihara, Kanagawa, 252-5258, Japan

d) Department of Chemistry, Hiroshima University, Higashi-Hiroshima, Hiroshima, 739-8526, Japan

e) Institute of Natural Science, Ural Federal University, Ekaterinburg, 620083, Russia

f) Division of Natural and Environmental Sciences, The Open University of Japan, Mihama, Chiba, 261-8586, Japan

\begin{abstract}
We microscopically investigate chiral magnetic orders in the absence and presence of magnetic field in a chiral magnetic crystal $\mathrm{CrNb}_{3} \mathrm{~S}_{6}$ by means of low-temperature Lorenz transmission electron microscopy and small-angle electron scattering method. Based on detailed analyses in both real and reciprocal space, we directly observe that chiral soliton lattice (CSL) emerges in small magnetic fields applied perpendicular to the chiral crystallographic axis. CSL develops from chiral helimagnetic structure (CHM) with increasing the spatial period from $48 \mathrm{~nm}$ toward sample size in rising magnetic fields. Chiral magnetic orders of CSL and CHM do not exhibit any structural dislocation, indicating their high stability and robustness. This is because chiral magnetic orders are macroscopically induced by monoaxial Dzyaloshinkii-Moriya exchange interaction that is allowed in hexagonal $\mathrm{CrNb}_{3} \mathrm{~S}_{6}$ crystals belonging to noncentrosymmetric chiral space group. Present observations of periodic, nonlinear, tunable, and robust CSL will be the first step to explore fascinating functions of CSL for magnetic and spintronic device applications using chiral magnets.
\end{abstract}

Key Words: Chiral soliton lattice, Chiral magnet, Lorentz transmission electron microscopy, Small-angle electron scattering

\section{INTRODUCTION}

Magnetic crystals with helical structures break mirror and inversion symmetries but combine rotational and translational symmetries. Namely, such magnetic crystals belong to noncentrosymmetric chiral space group. In these chiral magnetic crystals, orbital motions of localized electrons with spin magnetic moments take helical paths in the chiral framework of atoms and mediate coupling of the neighboring spins of electrons via the relativistic spin-orbit interaction called Dzyaloshinskii-Moriya (DM) interaction. This antisymmetric DM exchange competes with ferromagnetic (FM) exchange interaction in chiral magnetic crystals, which will result in an emergence of chiral magnetic orders (chiral configuration of spin magnetic moments) and various interesting functionalities unique to chiral magnets. In this respect, the concept of chirality, which means left- or right-handedness and plays an essential role in symmetry properties of nature at all length scales from elementary particles to biological systems, will be an important research strategy to explore a wide range of research fields of magnetism including nano-magnetism, spintronics, magneto-photonics, and so on. Furthermore, it will open up novel ways of device applications in related research fields.

In this study, we microscopically analyze magnetic structures of chiral magnetic orders that emerge in chiral magnetic $\mathrm{CrNb}_{3} \mathrm{~S}_{6}$ crystals in the absence and presence of small magnetic fields by means of low-temperature Lorenz transmission electron microscopy (TEM) and small-angle electron scattering (SAES) method. It is directly observed that chiral magnetic soliton lattice (CSL) develops in chiral helimagnetic structure (CHM) in rising magnetic fields perpendicular to the chiral crystallographic axis of $\mathrm{CrNb}_{3} \mathrm{~S}_{6}$ crystal. Furthermore, it is found that CHM and CSL are very stable against structural defects existing in crystals.

\section{EXPERIMENTAL}

Single crystals of $\mathrm{CrNb}_{3} \mathrm{~S}_{6}$ are grown in a gradient of temperature in a quartz tube by chemical vapor transport method. Several pieces of platelet single crystals grown in the same process of crystal growth are chosen to examine crystalline structures and magnetic properties by $X$-ray oscillation photography and magnetization measurements, respectively. Obtained data are consistent with those in literatures [1]. In these single crystals, the Curie temperature $T_{\mathrm{C}}$ is typically 123 
$\mathrm{K}$, determined by magnetization measurements.

For Lorentz TEM and SAES experiments, several thin specimens with typical thickness of $70 \mathrm{~nm}$ are fabricated by using focused Gallium-ion beams from $\mathrm{CrNb}_{3} \mathrm{~S}_{6}$ single crystals. Magnetic orders of CHM and CSL in $\mathrm{CrNb}_{3} \mathrm{~S}_{6}$ are visualized by means of Fresnel mode of Lorentz TEM. Specimens are mounted on a transmission electron microscope holder for specimen cooling, installed into a pole-piece of Lorentz lens or the objective lens in $200 \mathrm{kV}$ TEM machine; JEM2010M or JEM2010, respectively. Then, specimens are cooled down to $110 \mathrm{~K}$ below $T_{\mathrm{C}}$ with or without applying the magnetic field. The strength of magnetic field can be altered up to around $0.6 \mathrm{~T}$. These experimental setups enable to analyze detailed magnetic structures of CHM and CSL at various magnetic fields. In addition, characteristic magnetic structures of CHM and CSL (e.g., spatial modulation of spin configuration) are quantitatively analyzed in the reciprocal space by performing SAES experiments [2].

\section{RESULTS and DISCUSSION}

Figure 1 shows Lorentz micrographs of CHM in zero magnetic field and CSL at $0.208 \mathrm{~T}$. Figs. 1(a) and 1(c) are taken at an under-focused condition of $\delta f=-1158 \mathrm{~nm}$, while Figs. 1(b) and 1(d) at an over-focused condition of $\delta f=900 \mathrm{~nm}$. Black and white lines are provided for a guide to the eye to recognize that the contrast is reversed when defocusing in the opposite direction. From detailed analyses of the contrast profile in Lorentz micrographs as well as SAES reciprocal data, magnetic structures observed at zero magnetic field in Figs. 1(a) and 1(b) are found to exhibit a sinusoidal modulation of in-plane magnetic components with a single spatial frequency of $(48 \mathrm{~nm})^{-1}$. Namely, it is quite consistent with the magnetic structure of CHM, which should be a linear and harmonic configuration of spin magnetic moments.

With applying magnetic field perpendicular to the chiral crystallographic axis (c-axis), it is found that CSL emerges in CHM as shown in Figs. 1(c) and 1(d). Red arrows in Figs. 1(c) and 1(d) indicate the same magnetic soliton kink, i.e., 360 degree rotation of spin magnetic moments, in CSL. Apparently, CSL is a nonlinear and periodic magnetic structure. Indeed, although it is not shown in the present paper, magnetic scattering spots due to higher harmonic orders of CSL are observed in SAES data of CSL.

Incommensurate CSL undergoes a continuous phase transition to commensurate forced FM state at the critical field strength (about $0.23 \mathrm{~T}$ in the present specimen). Importantly, it is found that, upon applying magnetic field, CSL exhibits a continuous growth of the spatial period from $48 \mathrm{~nm}$ toward sample size at the incommensurate-to-commensurate (IC-C) phase transition. CSL is expected to work as the effective magnetic superlattice potential for the itinerant quantum spins. Importantly, the period of magnetic superlattice potential can be tuned by simply changing the strength of applied magnetic field. This is one of the most significant features of CSL.

Regarding magnetic chirality of CHM and CSL, it is identified to be left handed in the present crystals, based on detailed analysis of the contrast profile of CSL in Lorentz micrographs (i.e., an examination of the contrast profile in Figs. 1(c) and (d)). In the case of left-handed CSL, it should be observed as a dark (bright) contrast line at the center in an under(over-)focused condition, which is quite consist with the results observed in Fig. 1(c).

Figure 2(a) shows an in-focus TEM micrograph taken at room temperature. Broad streak patterns with bright contrast (indicated by red arrows) are found along [001] direction and remain to be observed above $T_{\mathrm{C}}$. Thus, these streak patterns correspond to scratch defects artificially made by an irradiation of focused Gallium-ion beams along [001] direction during the fabrication of TEM specimen. Similar scratch defects are found all over the specimen.

Figure 2(b) is a Lorentz micrograph of CHM taken in the same field of view as that in Fig. 2(a) at $110 \mathrm{~K} . \delta f$ is $-380 \mathrm{~nm}$. Importantly, stripe pattern of CHM appears very strict along [110] direction irrespective of these scratched defects. Similar behavior is observed in the case of CSL (e.g., Fig. 1(c)). In other words, CHM and CSL are very straight and appear very regularly perpendicular to the chiral $c$ axis almost all over the specimen, as partially shown in Figs. 1 and 2. As well, CHM and CSL present no structural dislocation and persist against crystal defects that exist in specimens and scratch defects introduced artificially. These results strongly suggest the extreme stability and robustness of CHM and CSL. This is because they are macroscopically induced by the monoaxial DM exchange interaction that is allowed in hexagonal $\mathrm{CrNb}_{3} \mathrm{~S}_{6}$ crystals belonging to $P 6_{3} 22$. Namely, CHM and CSL are macroscopic order of spin magnetic moments and extremely robust ground states regarded as topological spin phase object. 

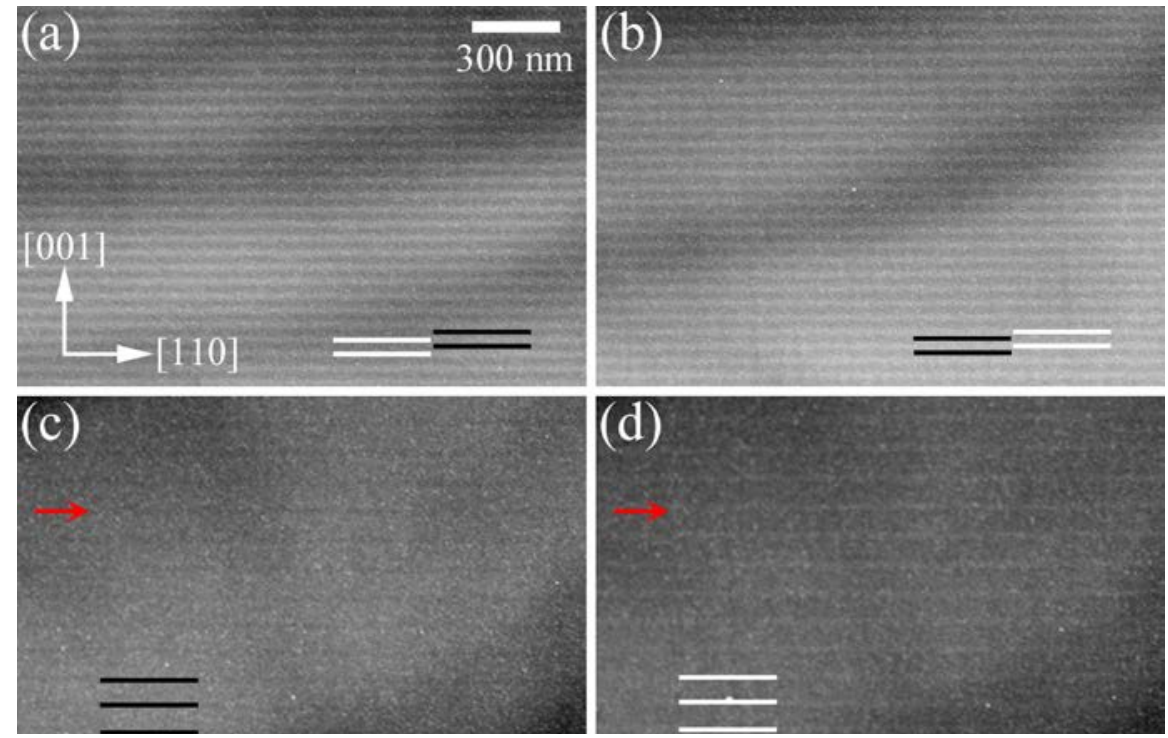

Fig. 1 Lorentz micrographs of CHM in zero magnetic field in (a) and (b) and CSL at $0.208 \mathrm{~T}$ in (c) and (d). (a, c) An under-focused Lorentz micrograph at a defocused value $\delta f=-1158 \mathrm{~nm}$. (b, d) An over-focused Lorentz micrograph at $\delta f=900 \mathrm{~nm}$. Black and white lines are given for a guide to the eye to recognize the reversal of the contrast in defocusing oppositely. Red arrows in (c) and (d) indicate the same magnetic soliton kink in CSL. All stripe fringes in (c) appear as dark contrast lines, meaning that CSL has left-handed magnetic chirality.
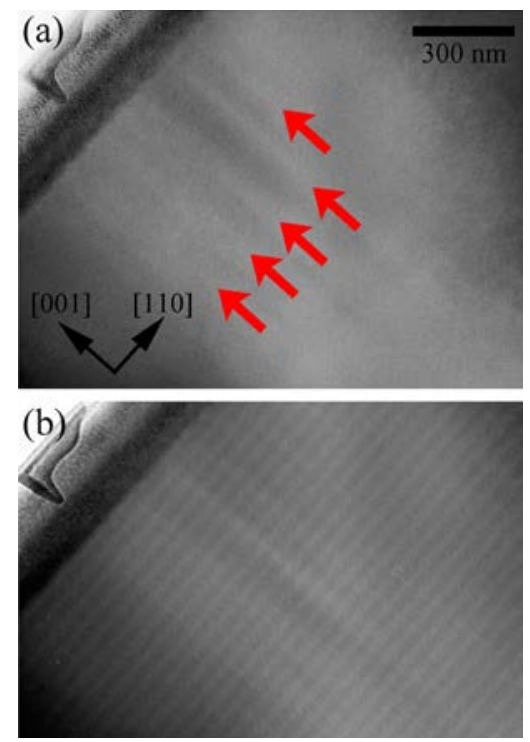

Fig. 2 Stability and robustness of chiral magnetic orders against scratch defect. (a) Infocused TEM micrograph at $300 \mathrm{~K}$. (b) Lorentz micrograph at 110 K. Stripe pattern of CHM appears very strictly perpendicular to the chiral $c$ axis.

\section{SUMMARY}

We directly present the formation of CSL in small magnetic fields in chiral magnetic crystals of $\mathrm{CrNb}_{3} \mathrm{~S}_{6}$. CSL develops in $\mathrm{CHM}$ in rising magnetic fields perpendicular to the chiral crystallographic axis. Furthermore, it is shown that CHM and CSL are very stable and robust against any perturbation existing in crystals. From theoretical viewpoints, CSL would exhibit a variety of interesting functions including spin current induction, nontrivial soliton transport [3], anomalous magneto-resistance [4], current-driven collective transport [5] and so on. Present observations will be the first step to explore these functionalities of CSL for spintronic device applications using chiral magnetic crystals.

\section{ACKNOWLEDGMENTS}

This study was partly supported by the Strategic Information and Communications R\&D Promotion Programme (SCOPE) by Ministry of Internal Affairs and Communications of Japan, Grant-in-Aid for Young Scientists (A) (No. 22686004).

\section{REFERENCES}

[1] Y. Togawa, T. Koyama, S. Mori, Y. Kousaka, J. Akimitsu, S. Nishihara, K. Inoue, A. S. Ovchinnikov and J. Kishine, Phys. Rev. Lett. 108, 107202 (2012).

[2] Y. Togawa, Microscopy, in press (2013); K. Takayanagi, T. Koyama, S. Mori, K. Harada and Y. Togawa, J. Electron Microscopy 61, 401 (2012); T. Koyama, K. Takayanagi, Y. Togawa, S. Mori and K. Harada, AIP Advances 2, 012195 (2012).

[3] Y. Togawa, Y. Kousaka, S. Nishihara, K. Inoue, J. Akimitsu, A. S. Ovchinnikov and J. Kishine, submitted.

[4] J. Kishine, I. V. Proskurin and A. S. Ovchinnikov, Phys. Rev. Lett. 107, 017205 (2011).

[5] J. Kishine, I. G. Bostrem, A. S. Ovchinnikov and VI. E. Sinitsyn, Phys. Rev. B 86, 214426 (2012).

[6] C. Pappas, Physics, 5, 28 (2012). 